\title{
Die „nicht mehr ganz so große“ Kronzeugenregelung
}

\author{
- Anmerkungen zum 46. Strafrechtsänderungsgesetz und zur Auslegung der \\ Konnexität im Rahmen des neugefassten $\S 46$ b StGB -
}

\section{Summary}

This aid to comprehending and prevention has been well known within the legal system of the Federal Republic of Germany for a long time. It has been used to date to its widest extent within the "principal witness regulation" of $\$ 46 \mathrm{~b}$ of the Criminal Code (StGB). It endowed courts with the power to afford offenders mitigation or dispensation in sentencing even if the actions revealed by them had no connection which those they had themselves undertaken.

After what was less than four years, the standard was subjected to reform via the 46th Criminal Justice Amending Law. By introducing the necessity for there being coherence between an act of serving as a principal witness and that of providing clarification, the broad field of application of the regulation has been narrowed. In this way, the legislative body wished, among other considerations, to afford greater significance to the principle of guilt predominating in the assessment of penalty and the level of trust invested by the population in the inviolability of the legal system.

The present piece deals critically with the reform of $\$ 46 \mathrm{~b}$ of the Criminal Code $(\mathrm{St} G B)$ and of the definition of the term of coherence. To this end, the concept of coherence is examined in terms of its definition to date before the reform is subjected to a critical appraisal in terms of its effectiveness and implementation. The attempt is then made to arrive at a distinct interpretation of the requirement for coherence according to $\$ 46$ b Criminal Code (StGB).

\section{Résumé}

La procédure de coopération des contrevenants à l'élucidation et à la prévention de délits fait depuis longtemps partie du système juridique de la République fédérale d'Allemagne. Avec l'instauration de la loi sur les repentis ("Kronzengenregelung ») au $\S 46 b$ du Code pénal, elle a acquis une portée sans précédent. Cette réglementation permettait aux tribunaux d'accorder aux délinquants une atténuation, voire une exemption de peine même lorsqu'il n'existait aucun rapport entre les faits divulgués et les délits qu'ils avaient eux-mêmes commis.

Quatre années à peine après son introduction, cette réglementation a été réformée par la $46^{\mathrm{e}}$ loi portant modification du droit pénal. L'instauration d'une connexité obligatoire entre le repentir et l'élucidation des faits a restreint le vaste champ d'application de la réglementation. Ce faisant, le législateur entendait notamment mieux tenir 
compte du principe de culpabilité prévalent dans la fixation de la peine et de la confiance de la population dans le caractère inébranlable de l'ordre juridique.

Le présent article porte un regard critique sur la réforme du $\$ 46 \mathrm{~b} d u$ Code pénal et sur la définition de la notion de connexité. À cet égard, il analyse ladite notion dans sa forme actuelle, avant de procéder à une évaluation critique de la réforme en regard de son efficacité et de sa mise en ouvre. Pour finir, il tente de présenter une interprétation propre, conformément au $\$ 46 \mathrm{~b}$ du Code pénal, de l'exigence relative au rapport entre les faits divulgués et les faits commis.

\section{Einleitung}

Mit Gesetz vom 29. Juli 2009 trat zum 1. September desselben Jahres die „Kronzeugenregelung" des $\S 46 \mathrm{~b}$ StGB in Kraft. ${ }^{1}$ Diese ermöglicht es den Gerichten, einem Straftäter, der zur Aufklärung oder Prävention eines Delikts beiträgt, Strafmilderungen oder - in bestimmten Fällen - sogar Straffreiheit zu gewähren. Der Einzug dieser Norm in das Strafgesetzbuch wurde von teilweise heftiger Kritik begleitet. ${ }^{2}$ Dabei war die Figur des Aufklärungs- und Präventionsgehilfen ${ }^{3}$ zu diesem Zeitpunkt für das deutsche Recht keineswegs mehr neu. Mit den $\S \S 129,129$ a und 261 X a.F. StGB ${ }^{4}$ sowie den im Jahre 1999 ausgelaufenen Art. 4, 5 Kronzeugengesetz (KronzG) $)^{5}$ und $\S 31$ BtMG kannte unsere Rechtsordnung bereits bereichsspezifisch geregelte Fälle der Aufklärungshilfe, wobei insbesondere Letztgenannter sich zu einem reichlich genutzten Werkzeug der Strafverfolgungsbehörden entwickelt hat. ${ }^{6}$

Der Gesetzgeber hat mit $\S 46$ b StGB allerdings in zweierlei Hinsicht Neuland betreten: Zum einen erschien die Aufklärungshilfe erstmals im allgemeinen Teil des StGB und erfuhr dadurch als besonders herausgehobene Form des positiven Nachtatverhaltens eine gewisse - aufgrund der kriminalpolitischen Ambivalenz von Kronzeugenregelungen für viele fragwürdige - ,Adelung“. Zum anderen wurde erstmals eine allgemeine

1 Dreiundvierzigstes Gesetz zur Änderung des Strafgesetzbuches - Strafzumessung bei Aufklärungs- und Präventionshilfe, BGB1. 2009 I, S. 2288; BT-Drs. 16/6268.

2 Vgl. Deutscher Anwaltverein, Stellungnahme Nr. 39/2006; Frank/Titz, ZRP 2009, 137; König, NJW 2009, 2481; Malek, StV 2010, 200; Salditt, StV 2009, 375.

3 Wenn nachfolgend von Aufklärungshilfe gesprochen wird, so ist damit auch die Präventionshilfe gemeint.

$4 \S 261$ X StGB wurde durch Art. 1 Nr. 5 des 43. StrÄndG mit Einführung des $\S 46$ b StGB aufgehoben.

5 Gesetz zur Änderung des Strafgesetzbuches, der Strafprozeßordnung und des Versammlungsgesetzes und zur Einführung einer Kronzeugenregelung bei terroristischen Straftaten vom 9. Juni 1989 (BGBl. 1989 I, S. 1059), ergänzt durch Art. 5 des Gesetzes zur Änderung des Strafgesetzbuches, der Strafprozeßordnung und anderer Gesetze (Verbrechensbekämpfungsgesetz) vom 28. Oktober 1994 (BGBl. 1994 I, S. 3186).

6 Quentin, Festschrift für Stöckel, 2010, 463 (463). Mühlhoff/Pfeiffer, ZRP 2000, 121 (123) berichten von 6.100 Fällen, die allein im Bundeszentralregister zwischen 1982 und 1998 aufgenommen wurden. 1995 soll § $31 \mathrm{Nr} .1$ BtMG in jedem zweiten BtM-Verfahren zur Anwendung gekommen sein, vgl. Jeßberger, Kooperation und Strafzumessung, 1999, S. 78 m.w.N. Zum Vergleich: Dem Bundeszentralregister wurden bzgl. § 46 b StGB bislang 389 Anwendungsfälle gemeldet (Stand: 8. Juli 2013). 
Strafzumessungsnorm für die Aufklärungshilfe vorgesehen, die sich nicht mehr auf bestimmte, strikt abgegrenzte Deliktsbereiche beschränkte, sondern die mit ihrem ausgesprochen weiten Anwendungsbereich Straftaten aus verschiedensten Kriminalitätskategorien umfasste. Besonders bemerkenswert war dabei, dass es für eine mildere Bestrafung bereits genügen sollte, dass der Täter Aufklärungshilfe hinsichtlich irgendeiner geeigneten Straftat leistet, ohne dass die offenbarte Tat einen Zusammenhang zu seiner eigenen aufweisen musste, was - nach den Worten von Kaspar/Wengenroth ${ }^{7}-\S 46 \mathrm{~b}$ StGB im Vergleich zu den bisherigen „kleinen“ zu einer ,ganz großen“ Kronzeugenregelung mache. Das Fehlen einer notwendigen Konnexität zwischen Kronzeugen- und Aufklärungstat und die dadurch hervorgerufene Weite der Norm wurden von Anfang an äußerst skeptisch betrachtet. ${ }^{8}$

Schon in ihrem Koalitionsvertrag hatten sich CDU/CSU und FDP auf eine Begrenzung des Anwendungsbereichs durch Aufnahme eines entsprechenden Konnexitätserfordernisses in den Tatbestand des $\S 46$ b StGB geeinigt. ${ }^{9}$ Kurz vor Ablauf der Legislaturperiode und unmittelbar vor dem vierten Geburtstag der Norm wurde dieses Vorhaben mit dem 46. Strafrechtsänderungsgesetz ${ }^{10}$ in die Tat umgesetzt und schien in Politik, Wissenschaft und Praxis auf breite Zustimmung zu stoßen. Tatsächlich aber wirft die Neufassung des $\S 46$ b StGB einige Fragen auf, weshalb man sich mit allzu voreiligen Lobeshymnen zurückhalten sollte. Bevor jedoch auf die Kritikpunkte im Einzelnen eingegangen wird, lohnt es sich zunächst, sich mit dem Konnexitätsprinzip in seiner bisherigen Ausprägung kurz auseinanderzusetzen.

\section{Zur bisherigen Auslegung des Konnexitätsprinzips}

Der Anwendungsbereich des $\S 46 \mathrm{~b}$ StGB ist, wie schon in seiner ursprünglichen Fassung, eröffnet, wenn es sich bei der Kronzeugentat um eine Straftat handelt, die mit einer im Mindestmaß erhöhten oder mit lebenslanger Freiheitsstrafe bedroht ist und die vom Aufklärungsgehilfen offenbarte Tat im Katalog des $\S 100$ a II StPO enthalten ist. Aus dem Wortlaut des $\S 46$ b I 1 StGB ergibt sich also, dass der Zusammenhang zwischen der Tat des Kronzeugen und der aufgeklärten Tat nicht dahingehend missverstanden werden darf, dass die beiden Straftaten identischen Kriminalitäts- oder Deliktsfeldern entspringen müssen. ${ }^{11}$

7 Kaspar/Wengenroth, GA 2010, 453 (454).

8 Vgl. Frank/Titz, ZRP 2009, 137 (139); Sahan/Berndt, BB 2010, 647 (648); Salditt, StV 2009, 375 (376).

9 Die entsprechende Vereinbarung findet sich auf S. 107 des Koalitionsvertrages.

10 Sechsundvierzigstes Strafrechtsänderungsgesetz - Beschränkung der Möglichkeit zur Strafmilderung bei Aufklärungs- und Präventionshilfe (46. StrÄndG) vom 10. Juni 2013, BGB1. 2013 I, S. 1497. Das Gesetz ändert in Art. 2 auch die Bestimmungen in § 31 BtMG und passt diese an die Neuregelung des $\S 46 \mathrm{~b}$ StGB an. Insbesondere sollen hierdurch ungewollte Umkehrschlüsse vermieden werden, die durch das Fehlen eines ausdrücklich geregelten $\mathrm{Zu}$ sammenhangserfordernisses in $\S 31$ BtMG hätten entstehen können. Andere Neuerungen in $\S 31$ BtMG haben lediglich Klarstellungsfunktion oder dienen der Festschreibung von seitens der Rechtsprechung bereits entwickelten Leitlinien zur Anwendung der Norm. Zu den Neuerungen vgl. BT-Drs. 17/9695, 9 sowie Peglau, NJW 2013, 1910 (1913).

11 BT-Drs. 17/9695, 7. 
Die künftige Beurteilung des Konnexes soll sich nach dem Willen des Gesetzgebers vielmehr an den vom Bundesgerichtshof festgelegten Leitlinien zu § 31 BtMG orientieren. ${ }^{12}$ Danach kann eine Konnexität nicht nur in Fällen gegeben sein, in denen der Täter an der offenbarten Tat selbst beteiligt war, sondern die Anwendung der Kronzeugenregelung soll auch dann möglich sein, wenn es sich bei Aufklärungs- und Kronzeugentat um rechtlich selbstständige Taten handelt, in die der mit den Behörden kooperierende Täter nicht unmittelbar involviert war oder die nicht Gegenstand der Anklage gegen den Aufklärungsgehilfen sind. ${ }^{13}$ Ergänzend verweist die Gesetzesbegründung für die Auslegung auf das ehemalige KronzG, welches in Art. $4 \S 1$ sowie in Art. 5 verlangte, dass es sich bei Kronzeugen- und Aufklärungstat um Taten nach $\S \S 129,129$ a StGB oder um mit diesen zusammenhängende Straftaten handeln muss. Hier wurde die Konnexität auf Taten erstreckt, auf deren Begehung die terroristische oder kriminelle Vereinigung gerichtet war oder die typische Begleitdelikte ihrer Mitglieder oder Unterstützer darstellten. ${ }^{14}$

Bezogen auf den nicht deliktsspezifisch ausgerichteten $\S 46 \mathrm{~b}$ StGB soll darauf abgestellt werden, ob die vom Kronzeugen begangene Tat (sowie die dabei verwirklichte Schuld) und die von ihm offenbarte Tat deshalb im Zusammenhang stehen, weil sie Teil eines kriminellen Gesamtgeschehens sind und sich in das verabredete Gesamtgeschehen einfügen, so dass sich ein innerer und inhaltlicher Bezug zwischen beiden Delikten feststellen lässt. ${ }^{15}$

Im Falle eines Zusammenschlusses mehrerer Personen zu einer Bande wird ein ausreichender innerer Bezug jedoch nicht allein durch die Mitgliedschaft in der kriminellen Gruppierung erreicht. Ebenso wenig genügt für die Bejahung der Konnexität ein zeitliches oder räumliches Zusammentreffen der beiden in Frage stehenden Delikte oder eine persönliche Beziehung zwischen Kronzeugen und Belastetem. ${ }^{16}$ Dagegen vermag die Förderung des Gruppenzwecks oder des Zusammenhalts in der Bande die nötige innere Verbindung zur eigenen Tat und Schuld des Kronzeugen herzustellen. ${ }^{17}$

Es kommt damit auf die Feststellung an, welche Ziele die jeweilige kriminelle Vereinigung verfolgt und welche Straftaten vom gemeinsamen Gesamtplan gedeckt sein sollen. Wenn also ein Mitglied einer Bande von gewerbsmäßigen Betrügern ein anderes Mitglied wegen einer schweren Körperverletzung belasten möchte, so ist der Wille der Bandenmitglieder, wie er im deliktischen „Gesellschaftsvertrag“ oder in der Planung für die konkrete Tat niedergelegt wurde, zu ermitteln. Sind Körperverletzungsdelikte nicht vom gemeinsam geplanten Geschehensablauf oder Bandenziel erfasst und handelt es sich auch nicht mehr um typische Begleitdelikte, so besteht zwischen offenbarter Tat und Kronzeugentat kein Zusammenhang, wodurch dem Aufklärungsgehilfen die Straf-

12 Dazu sowie zum Folgenden auch BT-Drs. 17/9695, $8 \mathrm{f}$.

13 BGH, StV 1985, 415 (416); BGH, StV 1995, 367; Körner/Patzak/Volkmer/Patzak, BtMG, $\S 31$ Rn. 61.

14 BT-Drs. 11/2834, 14; BT-Drs. 12/6853, 40.

15 BT-Drs. 17/9695, 8; Peglau, NJW 2013, 1910 (1912).

16 BT-Drs. 17/9695, 8.

17 Peglau, NJW 2013, 1910 (1912). 
milderung des $\S 46 \mathrm{~b}$ StGB verwehrt bleibt und er lediglich über die allgemeine Strafzumessungsnorm des $\S 46$ II StGB auf eine Straferleichterung hoffen kann. ${ }^{18}$

Wo kein Zusammenschluss mehrerer Personen besteht, sondern die Delikte aufeinander aufbauen, wird verlangt, dass die Tat des Aufklärungsgehilfen zumindest mittelbar die aufgeklärte Tat unterstützt hat oder dass umgekehrt die offenbarte Tat diejenige des Kronzeugen vorbereitet und abgesichert hat. ${ }^{19}$ Allerdings dürfte es noch nicht genügen, wenn mit der eigenen Tat lediglich eine durch einen anderen in strafbarer Weise herbeigeführte Situation ausgenutzt wird, sofern es an einer gemeinsamen verbindenden Zielsetzung der Beteiligten fehlt. Diese ist beispielsweise zwischen Dieb und Hehler aufgrund des gemeinsamen Verwertungsgedankens ebenso gegeben wie unter Mitgliedern einer Internetseite mit kinderpornografischen Inhalten, deren gemeinsames Interesse es ist, den Austausch und die Verbreitung des entsprechenden Materials zu fördern. ${ }^{20}$ Wird aber beispielsweise ein vom Vortäter bei einem Raub verletztes Opfer von einem anderen, der das Geschehen zufällig beobachtet hat, sexuell missbraucht, dann fehlt bei der späteren Offenbarung des Raubs durch den Sexualstraftäter ein hinreichend starkes verbindendes Element zur Tat des Kronzeugen, das die Bejahung der Konnexität rechtfertigen könnte. Andernfalls würde bei jedem Verhalten, das irgendwie an ein vorangegangenes strafbares Tun anknüpft, der Zusammenhang im Sinne des § 46b StGB gegeben sein, was dem eher auf Restriktion ausgerichteten Willen des Gesetzgebers zuwiderliefe.

\section{Die Reform in der Diskussion}

Nachdem der Zusammenhangsbegriff, wie er der Gesetzesbegründung zugrunde gelegen haben dürfte, dargestellt worden ist, soll nun zur Neufassung des $§ 46 \mathrm{~b}$ StGB kritisch Stellung genommen werden.

\section{Einschränkung des Anwendungsbereichs}

Zunächst stellt sich die Frage, ob mit der Änderung der Norm die vom Gesetzgeber intendierte Einschränkung des Anwendungsbereichs des $\S 46$ b StGB überhaupt erreicht wird.

Gemäß den Ausführungen Lückemanns in seiner Stellungnahme zum Reformentwurf ${ }^{21}$ spielt der externe Kronzeuge (bei dem eine Konnexität also nicht gegeben ist) in der Praxis eine marginale Rolle. ${ }^{22}$ Unter den bisherigen Verfahren vor dem Bundesge-

18 BT-Drs. 17/9695, 8. Weitere vertiefende Beispiele finden sich bei Peglau, NJW 2013, 1910 (1912).

19 BT-Drs. 17/9695, $8 \mathrm{f}$.

20 Hierzu auch Lückemann, Stellungnahme für die öffentliche Anhörung des Rechtsausschusses des Deutschen Bundestages am 12. Dezember 2012, S. 5; Peglau, NJW 2013, 1910 (1912).

21 Die zitierten Stellungnahmen zum 46. Strafrechtsänderungsgesetz im Rahmen der 108. Sitzung des Rechtsausschusses des Deutschen Bundestages vom 12.12.2012 können im Internet unter http://webarchiv.bundestag.de/cgi/show.php?fileToLoad=2930\&id=1223 (19.5.2014) abgerufen werden.

22 Lückemann, Stellungnahme, S. $1 \mathrm{f}$. 
richtshof finden sich nur wenige, in denen ein Zusammenhang zwischen Kronzeugenund Aufklärungstat fehlte. ${ }^{23}$ Mangels statistischer Erhebungen sind Aussagen über die tatsächliche Häufigkeit externer Kronzeugen allerdings kaum möglich. Wenn aber, wie Lückemann meint, der externe Kronzeuge der Ausnahmefall ist, so ist die einschränkende Wirkung des Zusammenhangserfordernisses tatsächlich begrenzt.

Hinzu kommt, dass sich die Reichweite der Konnexität gerade im Bereich der Organisierten Kriminalität und der Wirtschaftskriminalität sehr weit dehnen lässt. Die zu $\S 31$ BtMG entwickelten Grenzlinien der Rechtsprechung geben zwar einen ungefähren Leitfaden für die Rechtsanwendung an die Hand, gleichwohl dürfte die Bestimmung des Zusammenhangs zwischen Kronzeugen- und Aufklärungstat bei der Anwendung der BtMG-Norm aufgrund ihrer deliktsspezifischen Grundausrichtung einfacher sein als beim breiter angelegten $\S 46 \mathrm{~b}$ StGB. Im Bereich der Organisierten Kriminalität herrscht gerade in größeren Organisationen, die in unterschiedlichsten Bereichen tätig sind, eine hohe Diversität an in Frage kommenden Straftaten, was eine exakte Konturierung der Konnexität erschweren kann. Verschärft wird dieses Problem zusätzlich durch die Miteinbeziehung typischer Begleittaten, welche den Zusammenhangsradius erheblich erweitern.

\section{Zur besseren Legitimierbarkeit gegenüber dem Schuldprinzip}

Selbst wenn die vom Gesetzgeber bezweckte Einschränkung erreicht wird, fragt sich, ob diese tatsächlich notwendig ist. Der Gesetzgeber verweist darauf, dass, aufgrund des weiten Anwendungsbereichs des $\S 46 \mathrm{~b}$ StGB, die Norm nicht mehr im Einklang mit dem in $\S 46$ I 1 StGB geregelten Schuldprinzip stünde. ${ }^{24}$ So wird in der Begründung des Gesetzesentwurfs ausgeführt, dass durch Angaben des externen Kronzeugen die unmittelbare Tatschuld, wenn überhaupt, so nur mittelbar und in äußerst zweifelhafter Weise berührt sein könne. Trotz der Abwägungskriterien des $§ 46$ b II Nr. 2 StGB (der auch eine Berücksichtigung der Schwere der Schuld des Täters bei der Strafzumessung vorsieht), ermögliche die Kronzeugenregelung ohne ein einschränkendes Konnexitätskriterium unangemessene Strafmilderungen, die dem Prinzip der materiellen Gerechtigkeit zuwiderliefen und das Vertrauen der Opfer sowie der Bevölkerung in die Unverbrüchlichkeit der Rechtsordnung beeinträchtigen könnten. ${ }^{25}$

Natürlich bleibt ein bitterer Nachgeschmack, wenn ein hochgradig krimineller Delinquent für ein schweres Delikt eine relativ milde Strafe erhält. Man muss allerdings fragen, ob das Vertrauen der Bevölkerung oder der Opfer nicht auch dann beeinträchtigt werden kann, wenn ein Täter zwar zwei Jahre länger inhaftiert wird, der Staat im Gegenzug aber die Gelegenheit einer Aufklärungshilfe nicht ergreift, so dass die Komplizen oder andere Straftäter auf freiem Fuß bleiben und weitere Straftaten begehen kön-

23 Vgl. beispielsweise BGHSt 55, 153; BGH NStZ-RR 2013, 203.

24 BT-Drs. 17/9695, 6. Kritisch auch Salditt, StV 2009, 375 (376); Streng, Strafrechtliche Sanktionen, 2012, Rn. 597. Vgl. zu $\S 46$ b StGB und seinem Verhältnis zum Schuldprinzip im Allgemeinen Kaspar/Wengenroth, GA 2010, 453, (462 ff.); Kneba, Die Kronzeugenregelung des $\S 46$ b StGB, 2011, S. 122 ff. m.w.N.

25 BT-Drs. 17/9695, 6. Ebenso zu § 46b StGB allgemein Frank/Titz, ZRP 2009, 137 (139). 
nen. ${ }^{26}$ Zudem ist es nicht ausgeschlossen, dass ein Opfer trotz der Strafmilderung aus dem Urteilsspruch Befriedigung erlangen kann, wenn aus der Offenbarungshandlung des Angeklagten seine Reue und das Abstandnehmen von seiner kriminellen Vorgeschichte zum Ausdruck kommen. ${ }^{27}$

Darüber hinaus sorgt die Herausnahme des externen Kronzeugen aus $\S 46 \mathrm{~b}$ StGB und die Würdigung seines Aufklärungsbeitrages im Rahmen des $§ 46$ II StGB nicht zwingend für eine verbesserte Legitimierbarkeit mit Blick auf das Prinzip der materiellen Gerechtigkeit. Auch im Rahmen der allgemeinen Strafzumessung können, beispielsweise über die Einordnung einer Tat als minder schweren Fall, zum Teil deutliche Strafmilderungen erreicht werden, ${ }^{28}$ sodass die Vermittelbarkeit gegenüber Opfern und Bevölkerung problematisch werden kann, selbst wenn aus dem Aufklärungsverhalten Rückschlüsse auf einen verminderten Schuldvorwurf möglich sein sollten. Durch die Ausklammerung externer Kronzeugen wird das Legitimierungsproblem daher nicht gelöst, sondern höchstens abgeschwächt.

Was die Frage möglicher ungerechter Urteile betrifft, so sollte auch die Bedeutung des richterlichen Ermessens nicht außer Betracht bleiben. Durch entsprechendes Feingefühl bei der Beurteilung des Aufklärungserfolgs und der Strafmilderung kann den Interessen der Opfer Rechnung getragen werden und eine an Gerechtigkeitserwägungen ausgerichtete Strafzumessung stattfinden, die auch den Empfindungen der rechtstreuen Bevölkerung gerecht werden kann. Die Rolle der Gerichte bei der Anwendung der Kronzeugenregelung darf nicht einfach übergangen werden. ${ }^{29} \S 46 \mathrm{~b}$ StGB ist eine Kann-Vorschrift, die es dem Richter ermöglicht, im jeweiligen Einzelfall, auch unter Berücksichtigung der Schuld des Angeklagten ( $\$ 46$ b II Nr. 2 StGB), adäquat auf dessen Taten zu antworten und diese angemessen zu bestrafen. ${ }^{30}$ Zumindest sind bislang keine Fälle bekannt geworden, in denen Gerichte willkürlich anmutende, unverständlich niedrige Strafen als Gegenleistung für eine Aufklärungshilfe verhängt haben. ${ }^{31}$ Es besteht daher keine Veranlassung, aufgrund der bislang rein hypothetischen Möglichkeit schuldunangemessener Strafen der strafrichterlichen Abwägungsentscheidung zu misstrauen. ${ }^{32}$

26 Ebenso fragt sich, inwiefern das Vertrauen der Bevölkerung und der Opfer in die Unverbrüchlichkeit der Rechtsordnung durch die Begrenzung der Präventionshilfe (und damit der Möglichkeit der Verbrechensverhütung und des Rechtsgüterschutzes) gestärkt werden soll. Gegen eine Erstreckung des Konnexitätsprinzips auf die Präventionshilfe zu Recht Lückemann, Stellungnahme, S. 8 f. sowie Peglau, NJW 2013, 1910 (1911).

27 Auch wenn solche Fälle in der Praxis eher selten vorkommen mögen. Skeptisch hinsichtlich einer Beeinträchtigung des Rechtsempfindens ferner Kaspar, Stellungnahme für die öffentliche Anhörung des Rechtsausschusses des Deutschen Bundestages am 12. Dezember 2012, S. 4 ff.; Lückemann, Stellungnahme, S. 4; Peglau, NJW 2013, 1910 (1911).

28 Deutscher Anwaltverein, Stellungnahme Nr. 39/2006, S. 3, 6. Eine Ausnahme gilt hier natürlich bei zwingend vorgesehener lebenslanger Freiheitsstrafe.

29 Kaspar, Stellungnahme, S. $5 \mathrm{f}$.

30 Peglau, NJW 2013, 1910 (1911). So wurde im Verfahren LG Hamburg, 608 KLs 8/11 (bei juris) die Anwendung des $\S 46$ b StGB bei einem der Angeklagten unter anderem mit Blick auf die durch ihn verwirklichte Tatschuld abgelehnt.

31 Peglau, NJW 2013, 1910 (1911).

32 Ebenso Kaspar, Stellungnahme, S. 6. 
Wird mit Blick auf das Schuldprinzip ferner ausgeführt, dass durch die Reform des $\S 46$ b StGB dem Grundsatz schuldangemessenen Strafens dadurch noch stärker Rechnung getragen werden kann, dass derjenige, der ein mit der eigenen Tat in Zusammenhang stehendes Delikt offenbart, eher glaubhaft machen könne, dass er sich vom kriminellen Umfeld, in dem er seine eigene Tat begangen hat, lösen will, ${ }^{33}$ so kann auch dieser Begründungsansatz nicht überzeugen. Warum soll ein Mitglied einer Betrügerbande, das dabei hilft, durch andere Mitglieder begangene gewerbsmäßige Betrugsdelikte aufzuklären, an denen es selbst nicht unmittelbar beteiligt war, eine andere Einstellung zur seiner eigenen Tat haben, als ein Mitglied, das ein schweres Körperverletzungsdelikt aufklärt, welches anlässlich eines Betrugsversuches entgegen der vereinbarten Verfahrensweise der Gruppe von einem anderen Mitglied (deliktsuntypisch) verübt worden ist? ${ }^{34}$ Warum soll im einen Fall die innere Haltung des Aufklärungsgehilfen nobler sein als im anderen? Warum soll ein externer Kronzeuge, der, in Abkehr von seinem bisherigen Lebenswandel, eine sog. Lebensbeichte ablegt und umfassend zu ihm bekannten Straftaten aussagt, nicht in dem Maße belohnt werden können wie ein interner Kronzeuge, der aus Eigennutz ohne jede Reue handelt? Letzten Endes sagt die Konnexität nichts über die innere Einstellung des Täters aus, ${ }^{35}$ weshalb auch insofern die pauschale Herausnahme des externen Kronzeugen aus dem Anwendungsbereich des $\S 46 \mathrm{~b}$ StGB nicht hinreichend gerechtfertigt werden kann.

Ebenso unklar bleibt, warum der Ausschluss des externen Kronzeugen den Zweck des $\S 46$ b StGB - die Förderung des Aufbrechens geschlossener Täterkreise - stärker betonen können soll. Insofern argumentiert der Gesetzesentwurf, der interne Kronzeuge verfüge über die erforderliche Nähe zu den konspirativen Strukturen und dadurch über detaillierteres Wissen als ein externer Kronzeuge. Zugleich werde die Überprüfung der Glaubwürdigkeit erleichtert. ${ }^{36}$ Dies mag stimmen, wenn man den externen Kronzeugen als Person betrachtet, die stets gänzlich außerhalb der kriminellen Vereinigung steht und daher keinen umfassenden Einblick in die zugrundeliegenden Strukturen und Planungsvorgänge hat. Wie im obigen Betrügerbandenfall gezeigt, kann der externe Kronzeuge aber eben auch innerhalb der Organisation tätig sein und somit verlässlich von Straftaten anderer Mitglieder erfahren, die den Bereich der üblichen Verfahrensabrede unter den Bandenmitgliedern verlassen. Dass ein derartiger externer Kronzeuge weniger verlässliche Angaben zu anderen Straftaten machen können soll, leuchtet nicht ein. Ebenso können Mitglieder rivalisierender Gruppen weitreichende Erkenntnisse über die Vorgänge der jeweils anderen Bande haben (z.B. durch gegenseitige Kontakte im Milieu, gegenseitige Absprachen hinsichtlich der Verteilung der „Reviere“ oder durch Überläufer aus anderen Gruppen). Die Aussagekraft des Konnexitätsprinzips bezüglich der Glaubwürdigkeit des Kronzeugen ist daher ebenfalls begrenzt.

33 Vgl. dazu BT-Drs. 17/9695, 6.

34 S. zu diesem Beispiel auch BT-Drs. 17/9695, 8.

35 So auch Kaspar, Stellungnahme, 3.

36 BT-Drs. 17/9695, $6 \mathrm{f}$. 


\section{Nachteile bei der Rechtsanwendung}

Problematisch ist ferner, dass die bisherige Zielrichtung des Konnexitätsmerkmals in seiner Bedeutung durch die Reform geradezu in ihr Gegenteil verkehrt wird. ${ }^{37}$ Ursprünglich zielte die Einführung eines Zusammenhangs zwischen Kronzeugen- und Aufklärungstat darauf ab, den Anwendungsbereich des $\S 31$ BtMG zu erweitern. ${ }^{38}$ Nun aber soll sich dieses Extensionsinstrument zu einem Restriktionsmittel wandeln. Trotz des Verweises auf die bisherige BGH-Rechtsprechung zu $\S 31$ BtMG sollte deshalb nicht blind auf die dortigen Auslegungsmaximen zurückgegriffen werden, da mit der veränderten Zielrichtung des Zusammenhangserfordernisses auch eine Änderung der praktischen Handhabung der Konnexität durch die Rechtsprechung nicht gänzlich ausgeschlossen ist. ${ }^{39}$

Wenn der Gesetzgeber die künftige Ausgestaltung des Zusammenhangsbegriffs der Rechtsprechung überlässt, so führt dies in Verbindung mit der eben beschriebenen inhaltlichen Neuausrichtung zu nicht unerheblichen Problemen bei der Rechtsanwendung. Mit dem neuen Erfordernis der Konnexität wird im Rahmen des $\S 46$ b StGB nunmehr ein dritter Unsicherheitsfaktor geschaffen, der die Attraktivität der Norm schmälern dürfte. ${ }^{40}$ Schon bei der Altfassung konnte man kritisieren, dass aufgrund der schwer vorhersagbaren richterlichen Ermessensentscheidung hinsichtlich des Vorliegens eines ausreichenden Aufklärungserfolgs sowie dessen Würdigung im Rahmen der Strafzumessung dem potentiellen Kronzeugen kaum verlässliche Zusagen bezüglich der sanktionsbezogenen Auswirkungen einer Kooperation mit den Ermittlungsbehörden gemacht werden konnten. Hierdurch bestand eine nur schwache Verhandlungsgrundlage für die staatlichen Organe, denn der Beschuldigte war gezwungen in Vorleistung zu treten ohne Gewissheit zu haben, ob sich seine Mithilfe überhaupt strafmildernd auswirkt. ${ }^{41}$ Zusätzlich obliegt es nun der richterlichen Beurteilung, im Einzelfall zu entscheiden, ob die offenbarte Tat einen Zusammenhang zur Kronzeugentat aufweist. Solange sich die Rechtsprechung noch nicht klarstellend zu dieser Frage geäußert hat, ist daher festzuhalten, dass die Verhandlungsposition für Polizei und Staatsanwaltschaft bei der Anwendung des $\S 46 \mathrm{~b}$ StGB weiter geschwächt worden ist.

Ebenso kann für den kooperationsbereiten Beschuldigten die Neufassung der Norm zur Falle werden, da er in Grenzfällen nur schwer überschauen können wird, ob die Aufklärungstat tatsächlich in einem ausreichenden Zusammenhang zur eigenen Tat steht. Zu seiner ohnehin schon unsicheren Kosten-Nutzen-Rechnung kommt damit eine zusätzliche unbekannte Variable hinzu, wodurch für ihn der Anreiz zur Offenbarung

37 Zum Folgenden auch Lückemann, Stellungnahme, S. 2 ff.

38 Diese Tendenz zeigt sich bspw. in den Entscheidungen BGH, StV 1985, 415 (416); BGH, NJW 1987, 2882; BGH, StV 1995, 367. Zur "ausufernden Ausdehnung" des Anwendungsbereichs des $§ 31$ BtMG durch die Rechtsprechung s. Hassemer StV 1986, 550 (551); Körner, JR 1985, 427; Malek, Betäubungsmittelstrafrecht, 3. Aufl., Kap. 4 Rn. 15; Mühlhoff/ Pfeiffer, ZRP 2000, 121 (126).

39 Diese Möglichkeit sieht auch Lückemann, Stellungnahme, S. 3 f.

40 Hierzu auch Lückemann, Stellungnahme, S. 5.

41 Diese Problematik zeigte sich schon bei der Anwendung des ehemaligen KronzG, vgl. Mühlhoff/Pfeiffer, ZRP 2000, 121 (124). 
anderer Straftaten weiter geschmälert wird. ${ }^{42} \mathrm{Ob}$ der pauschale Hinweis darauf, dass notfalls über § 46 II StGB eine Strafmilderung erreicht werden könne (dessen Voraussetzungen aufgrund seiner Anbindung an das Schuldprinzip in $\S 46$ I 1 StGB gerade beim externen Kronzeugen allerdings auch nicht ohne weiteres erfüllt sein dürften), ${ }^{43}$ geeignet ist, die neue Unsicherheit aufzuwiegen, ist zu bezweifeln. Zumindest wird ihm noch mehr als schon zuvor anzuraten sein, sich von einem Rechtsbeistand frühzeitig intensiv beraten zu lassen, wobei die neue Gesetzeslage auch die Arbeit der Strafverteidiger nicht gerade erleichtern wird, solange keine eindeutigen Auslegungsrichtlinien von der Rechtsprechung herausgearbeitet worden sind. ${ }^{44}$

\section{Zur Umsetzung der Reform}

Auch hinsichtlich der Vorgehensweise ist die Reform kritikwürdig, wobei es eigentlich weniger darum geht, wie konkret vorgegangen wurde, sondern wie gerade nicht vorgegangen wurde.

Die Kritik bezieht sich maßgeblich auf die Tatsache, dass die Einschränkung des Tatbestandes aus Erwägungen erfolgte, die jedweder empirischen Grundlage entbehren. Hinsichtlich der oben erörterten Gefahr schuldunangemessener Strafen wird auf eine abstrakte Bedrohungslage abgestellt, anstatt anhand konkreter Beispiele darzulegen, wo und wie häufig solche frappant ungerechten Entscheidungen tatsächlich getroffen worden sind. Zudem wird die Behauptung aufgestellt, die Regelung in ihrer bisherigen Form verstoße gegen das Rechtsempfinden der Bevölkerung und der Opfer, ohne dies mit Fakten zu belegen. Tatsächlich verfügt man - soweit ersichtlich - über keinerlei Anhaltspunkte darüber, wie die Kronzeugenregelung von der Gesellschaft beurteilt wird. ${ }^{45}$ Es kann wohl davon ausgegangen werden, dass bei eher emotionsgeladenen Straftaten wie Sexual- und Tötungsdelikten mit einer relativ milden Bestrafung des Täters durchaus eine Erschütterung des Rechtsempfindens einhergehen kann. Ob die gleiche Empörung auch dann hervorgerufen wird, wenn opferlose Delikte oder bloße Eigentumsdelikte im Raume stehen, ist zweifelhaft, aber eben nicht empirisch belegt. Wenn die Befürworter der Neuregelung in ihren Ausführungen immer wieder auf die Bemerkung „Erfahrungen zeigen...“ ausweichen, so genügt dies nicht, um die Notwendigkeit der Begrenzung des $\S 46 \mathrm{~b}$ StGB ausreichend zu begründen. Es bleibt unklar, wessen Erfahrung hier zitiert wird, welche Quellen für die Meinungsbildung herangezogen werden und ob es sich wirklich um eine allgemeine Beobachtung oder schlichte Einzelfälle handelt oder die Informationen vom bloßen Hörensagen stammen.

42 Huth, Stellungnahme für die öffentliche Anhörung des Rechtsausschusses des Deutschen Bundestages am 12. Dezember 2012, S. 3.

43 Dazu Mushoff, KritV 2007, 366 (379); gegen eine Berücksichtigung bei fehlender Konnexität im Rahmen des positiven Nachtatverhaltens Steinberg, WuW 2006, 719 (723 f.).

$44 \mathrm{Zu}$ Problemen der Kronzeugenregelung aus Sicht der Strafverteidigung s. Malek, StV 2010, 200 ff.; Salditt, StV 2009, 375 (378 f.).

45 Kaspar, Gedächtnisschrift für Walter, 2014, S. 90; Middendorff, ZStW 85 (1973), $1102 \mathrm{ff}$. berichtet in seinen historischen Falldarstellungen aus Großbritannien zwar von sozialen Ächtungen oder der Tötung von Kronzeugen, doch dürften die dortigen Geschehnisse höchst begrenzte Rückschlüsse auf die heutige Einstellung der Bevölkerung zur vorliegenden Thematik zulassen. 
Die Verlässlichkeit dieser Erfahrungsberichte lässt sich anhand der Erörterungen im Rechtsausschuss des Bundestages beispielhaft veranschaulichen. So führte Glienke in seiner Stellungnahme sinngemäß aus, dass die Gefahr einer Falschaussage im Rahmen von $\S 46 \mathrm{~b}$ StGB vor allem unter dem Druck der Untersuchungshaft erhöht sei und der Inhaftierte in seiner Zwangslage auch nur beiläufig „Aufgeschnapptes“ an die Ermittlungsbehörden weitergeben würde, um sich aus seiner misslichen Lage befreien zu können. Hierdurch könne es zu einem regelrechten Handel mit nutzbringenden Informationen kommen. Stellenweise hätten sich bereits solche „Informationstauschbörsen“ entwickelt. ${ }^{46}$ Schneiderhahn hingegen stellte in seinen Ausführungen für den Deutschen Richterbund lediglich fest, dass zwar die Gefahr von Falschbelastungen durch externe Kronzeugen generell durchaus erhöht sei, dass ihm aber von Tauschbörsen bislang noch nichts berichtet worden sei. ${ }^{47}$ Welche der beiden Realitäten nun die richtige ist, lässt sich mangels aussagekräftiger Befunde nicht klären.

Das hier dargelegte Beispiel steht stellvertretend für sämtliche aufgeworfenen Reformgründe. Hinsichtlich der beschriebenen Gefahren und Nachteile, die vom externen Kronzeugen ausgehen, muss festgestellt werden, dass sämtliche Annahmen einen rein spekulativen Charakter haben, solange sie durch keine genaueren Untersuchungen verifiziert worden sind. Auf der Basis von allgemein gehaltenen Kritikpunkten und Hinweisen auf irgendwelche gemachten Erfahrungen lassen sich kriminalpolitische Entscheidungen nur schwer rechtfertigen. Zwar mag die genannte Reform nur einen relativ kleinen Teil der Aufklärungsgehilfen aus dem Anwendungsbereich des § 46b StGB ausschließen; die Vorgehensweise, die hier an den Tag gelegt wurde, bleibt enttäuschend, zumal sich für die Anwendung der Kronzeugenregelung eine empirische Evaluation problemlos angeboten hätte. ${ }^{48}$

\section{Zur möglichen Neuausrichtung des Konnexitätsbegriffs im Rahmen des $\S 46 b \mathrm{St} G B$}

Die geäußerte Kritik ändert gleichwohl nichts an der Tatsache, dass sich die Praxis mit der Neufassung des $\S 46$ b StGB auseinandersetzen muss. Wie schon erwähnt, hat der Gesetzgeber es der Rechtsprechung überlassen, die weiteren Konturen des Konnexitätsbegriffs zu bestimmen. Dass hier nicht ohne Weiteres auf die Rechtsprechung zu $\S 31$ BtMG zurückgegriffen werden sollte, wurde bereits erwähnt, soll aber anhand des bereits mehrfach bemühten Betrügerbandenfalles nochmals erläutert werden.

46 Glienke, Stellungnahme für die öffentliche Anhörung des Rechtsausschusses des Deutschen Bundestages am 12. Dezember 2012, S. 2.

47 Schneiderhahn, Stellungnahme für die öffentliche Anhörung des Rechtsausschusses des Deutschen Bundestages am 12. Dezember 2012.

48 Eine empirische Untersuchung vor Durchführung der Reform forderten auch Huth, Stellungnahme, S. 3, 13; Kaspar, Stellungnahme, S. 10. Der Bundesrat forderte bei der Einführung des $\S 46$ b StGB sogar eine Norm, die eine regelmäßige Evaluation vorschreiben sollte, vgl. BT-Drs. 16/6268, 18. Die Koalition aus CDU/CSU und SPD hat die Evaluierung der geltenden Kronzeugenregelungen im aktuellen Koalitionsvertrag ausdrücklich festgeschrieben (dort S. 102). An der Universität Augsburg wird ein entsprechendes Forschungsprojekt zur Anwendung der Kronzeugenregelung in der Rechtspraxis momentan durchgeführt. 
Nach derzeitigem Stand der Rechtsprechung unter Berücksichtigung der Auslegung des Zusammenhangsbegriffs im Rahmen des ehemaligen KronzG besteht bei Zusammenschlüssen mehrerer Personen zu einer Bande zwischen solchen Taten ein hinreichender Konnex, die im Rahmen der Gruppenvereinbarung liegen oder diese deliktstypisch flankieren. Gehört zum Vorgehen der Betrügerbande, dass deren Mitglieder zur Täuschung der Opfer falsche Dokumente verwenden, dann wird die dazugehörige Urkundenfälschung als Begleitdelikt unter das Konnexitätskriterium fallen. Konsequenterweise müsste es sogar genügen, wenn sich zwei für die Bande tätige Urkundenfälscher gegenseitig belasten, ohne dass es einer Beteiligung an den eigentlichen Betrugstaten bedürfte. Dies verdeutlicht wiederum, wie weit der Zusammenhangsbegriff verstanden werden kann.

Wenn sich nun aber die Tathandlung außerhalb des gruppenintern vereinbarten Geschehensablaufs bewegt und auch nicht als deliktstypisches Verhalten kategorisiert werden kann, dann ist der Konnex nach derzeitigem Auslegungsstand zu verneinen.

Dies ist in Fällen nachvollziehbar, bei denen die Handlung des Kronzeugen oder des Belasteten mit der Gruppenaktivität oder dem Gruppenzweck keinerlei Verbindungen aufweist. Das wäre im angeführten Beispiel der Fall, wenn einer der Betrüger ein anderes Mitglied wegen einer privat begangenen Steuerhinterziehung belasten würde. Zwar besteht hier eine Verbindung zwischen beiden Tätern aufgrund der Gruppenzugehörigkeit, sie wirkt sich im konkreten Handeln des Täters jedoch nicht aus und genügt für sich genommen ohnehin nicht für die Bejahung der Konnexität.

Wie liegen nun aber Fälle, in denen der Belastete oder der Kronzeuge im Rahmen ihrer Bandentätigkeit aktiv sind? Wenn zum Beispiel ein Bandenmitglied, bei dem der versuchte Betrug fehlschlägt, das Tatopfer ausnahmsweise niederschlägt, um doch noch an das Geld zu gelangen und damit das eigentliche Ziel der Bande zu erreichen, dann müsste für den Täter das Tor zu $§ 46 \mathrm{~b}$ StGB im Falle der Belastung eines anderen Bandenmitglieds verschlossen bleiben, wenn sich dieses Verhalten nicht im Rahmen des vereinbarten Geschehensablaufs bewegt oder den typischen Begleittaten zuzuordnen ist.

Dieses Ergebnis ist allerdings sehr unbefriedigend, gerade wenn man die Konstellation mit Fällen vergleicht, in denen lediglich ein typisches Begleitdelikt verwirklicht wurde und der Täter am gewerbsmäßigen Betrug als solchem nicht beteiligt gewesen ist. In dieser Grenzzone des Anwendungsbereichs der Kronzeugenregelung fragt sich, welcher Unterschied zwischen den beiden beschriebenen Fallkonstellationen eine unterschiedliche rechtliche Würdigung rechtfertigt. In beiden Fällen werden der Zusammenhalt der Gruppe und deren Ziele gefördert, so dass sich eine Beziehung zur eigenen Schuld des Kronzeugen durchaus herstellen ließe. Auch hinsichtlich der Gefahr von Falschbelastungen, des Umfangs des Insiderwissens des Aufklärungsgehilfen oder mit Blick auf die innere Einstellung des Aufklärungsgehilfen zur Tat lassen sich zwischen den beiden Fallgestaltungen keine grundlegenden Unterschiede erkennen, wie in den obigen Ausführungen zum Teil schon angedeutet wurde. ${ }^{49}$

49 Daher ist die Unbedenklichkeit der Einschränkung des § 46b StGB hinsichtlich des allgemeinen Gleichheitssatzes gemäß Art. 3 I GG (dazu BT-Drs. 17/9695, 7) gerade in Fällen wie dem hier verwendeten Betrügerbandenfall durchaus anzuzweifeln. 
Allenfalls mit Blick auf das Rechtsempfinden können sich Unterschiede ergeben, wenn beispielsweise die Tat des Aufklärungsgehilfen ein Mord ist und er sodann einen anderen wegen gewerbsmäßigen Betrugs belasten will. Hier erscheint es unbillig, dem Mörder einen weitreichenden Strafnachlass zu gewähren, da er ein vergleichsweise geringwertiges Delikt offenbart hat. Verneint man hier aber die Konnexität, dann muss man auch umgekehrt dem Bandenmitglied, das bei der Aufklärung eines Mordes mitgeholfen hat, die Milderungen nach $\S 46 \mathrm{~b}$ StGB verwehren. Hier fällt die Verneinung der Kronzeugenregelung nicht so eindeutig aus, wenn man allein auf das Rechtsempfinden abstellt. Dies spricht dafür, diesen Gesichtspunkt nicht bereits bei der Beurteilung des Zusammenhangs zwischen Kronzeugen- und Aufklärungstat heranzuziehen, sondern Unterschiede insofern erst im Rahmen der Strafzumessung zu würdigen.

Die Rechtsprechung wird früher oder später mit Fällen innerhalb der eben beschriebenen Grauzone konfrontiert werden ${ }^{50}$ und gerade hier wird sich auch die vom Gesetzgeber aufgetragene Konkretisierung der Zusammenhangskriterien abspielen. Um Wertungswidersprüche zu vermeiden, könnte man für die dargestellte Problematik zwei mögliche Auslegungswege beschreiten:

Die erste (restriktive) Auslegungsmöglichkeit könnte in der zurückhaltenden Einbeziehung der typischen Begleitdelikte in den Zusammenhangsradius bestehen.

Die zweite (extensive) Möglichkeit besteht in der Erweiterung des Zusammenhangsbegriffs über die Kategorie der typischen Begleittaten hinaus, um auch solche Delikte unter den Anwendungsbereich der Kronzeugenregelung zu fassen, die zwar außerhalb des vereinbarten Gesamtgeschehens und der Deliktstypik liegen, ausgehend von ihrer Zielrichtung aber auf die Förderung des Gruppenzwecks gerichtet waren. Man könnte demzufolge die Konnexität wie folgt verstehen:

Ein Zusammenhang zwischen Kronzeugen- und Aufklärungstat liegt nicht nur dann vor, wenn sich beide Taten im Rahmen des vereinbarten Geschehensablaufs abspielen oder sich als typische Begleittaten darstellen, sondern schon dann, wenn sie mit dem vereinbarten Ziel oder Zweck der Gruppe verbunden sind. Letzteres ist nicht schon dann der Fall, wenn Kronzeuge und Belasteter Mitglieder derselben Gruppe sind oder in einer persönlichen Beziehung zueinander stehen, sondern wenn sie die jeweiligen Taten anlässlich oder bei Gelegenheit einer Gruppenaktivität objektiv und subjektiv ,als Mitglieder" begangen haben, ihr Verhalten folglich nach dem äußeren Erscheinungsbild des Gesamtgeschehens und der inneren Willensrichtung letztlich der Förderung oder Verwirklichung des Gruppenzwecks diente.

Hierdurch werden auch bestimmte deliktsuntypische Handlungsweisen gegebenenfalls vom Konnexitätsbegriff erfasst, es bleiben aber Taten vom Anwendungsbereich ausgenommen, die mit der Tätigkeit der kriminellen Vereinigung keinerlei innere und äußere Verbindung aufweisen. ${ }^{51}$ Diese Auslegung kompensiert auch die fehlende Deliktsspezifität des $\S 46$ b StGB im Vergleich zu $\S 31$ BtMG, da sich auch bei letzterem im Grunde alle Delikte, die im Zusammenhang mit der Kronzeugentat stehen konnten, auf die Verfolgung eines gemeinsamen (Grund-)Zwecks, nämlich die Einfuhr und Ver-

50 Die beschriebenen Probleme können sich nicht nur im Rahmen krimineller Personenvereinigungen zeigen, sondern stellen sich ebenso bei aufeinander aufbauenden Delikten.

51 Für aufeinander aufbauende Straftaten kann es bei der bisherigen Auslegung bleiben, sofern das bloße Ausnutzen einer durch einen Vortäter geschaffenen Tatsituation - aufgrund der oben angeführten Erwägungen - aus dem Konnexitätsbereich ausgeklammert bleibt. 
breitung von Betäubungsmitteln zurückführen lassen. Eine extensive Auslegungsvariante erscheint somit durchaus vertretbar.

\section{Fazit}

Die Kronzeugenregelung des $\S 46 \mathrm{~b}$ StGB, dies ist zu betonen, birgt Gefahren in sich, vor allem mit Blick auf die Wahrung des Schuldprinzips. Anhand der vorstehenden Ausführungen wurde aber gezeigt, dass mit dem pauschalen Ausschluss externer Kronzeugen die Zahl der Probleme nicht unbedingt abnimmt. Die Reform dürfte zu früh kommen: einerseits fehlt es an einer ausreichend fundierten Begründung, andererseits haben sich in der Rechtsprechung noch keine festen Anwendungslinien herausbilden können, anhand derer ein Änderungsbedarf hätte ermittelt werden können. ${ }^{52}$

Ob die mit der Reform verbundenen Erwartungen erfüllt werden, bleibt abzuwarten. Bei Tätern, die andere bezüglich Taten belasten, die in keinerlei Zusammenhang mit der eigenen Tat oder den Handlungen der eigenen Gruppe stehen, mag die Einschränkung tatsächlich sinnvoll sein. Legt man aber den bisherigen Konnexitätsbegriff der Rechtsprechung der Neuregelung zugrunde, so kann es zu Differenzierungen zwischen internem und externem Kronzeugen kommen, die nur schwer nachzuvollziehen sind. Der bisherige Zusammenhangsbegriff wird daher sicherlich früher oder später an die Besonderheiten des $\S 46$ b StGB angepasst werden müssen. Die hier vorgestellten Ideen verstehen sich als Denkanstoß, um diese für die Zukunft der „Kronzeugenregelung“ wichtige Diskussion in Gang zu bringen.

52 So auch Lückemann, Stellungnahme, S. 2. Skeptisch bzgl. der Notwendigkeit der Reform auch Peglau, NJW 2013, 1910 (1913). 